\title{
Associação entre características de desempenho de tilápia-do-nilo ao longo do período de cultivo
}

\author{
Grazyella Massako Yoshida(1), Carlos Antonio Lopes de Oliveira(1), Sheila Nogueira de Oliveira(1), \\ Natalí Miwa Kunita ${ }^{(1)}$, Emiko Kawakami de Resende(2), Luiz Alexandre Filho(1) e Ricardo Pereira Ribeiro(1) \\ (1)Universidade Estadual de Maringá, Departamento de Zootecnia, Avenida Colombo, № 5.790, Jardim Universitário, CEP 87020-900, \\ Maringá, PR. E-mail: grazyoshida@hotmail.com, caloliveira@uem.br, she_uem@hotmail.com, nat_kunita@hotmail.com, lafilho@uem.br, \\ rpribeiro@uem.br (2)Embrapa Pantanal, Rua 21 de Setembro, no 1.880, CEP 79320-900, Corumbá, MS. E-mail: emiko@cpap.embrapa.br
}

Resumo - O objetivo deste trabalho foi estimar as herdabilidades e a estrutura de correlações genéticas entre as características de desempenho de tilápia-do-nilo (Oreochromis niloticus) da linhagem GIFT, em diferentes estágios do ciclo de produção. As tilápias foram cultivadas em tanques-rede. Mediu-se ganho em peso diário total, peso vivo e ganho em peso diário, em quatro períodos, com intervalos de aproximadamente 30 dias. Foram realizadas análises unicaracter para as medidas, em todas as biometrias e, nas análises bicaracter, as medidas de mesma característica foram combinadas duas a duas e com o ganho em peso diário total. As estimações de herdabilidade variaram de 0,15 a 0,11 para peso vivo, 0,16 a 0,09 para ganho em peso diário e 0,17 a 0,12 para ganho em peso diário total, nas análises unicaracter. Os valores estimados de correlação genética para peso vivo e ganho em peso diário, associados ao ganho em peso diário total, variaram entre 0,37 a 0,98 e 0,74 a 0,88 , respectivamente. A forte associação genética estimada entre peso vivo em biometrias intermediárias e ganho em peso diário total sugere que a seleção para velocidade de crescimento pode ser realizada de forma precoce.

Termos para indexação: Oreochromis niloticus, correlações genéticas, herdabilidade, linhagem GIFT, velocidade de crescimento.

\section{Association between performance traits of Nile tilapia during the culture period}

\begin{abstract}
The objective of this work was to estimate the structure and heritability of genetic correlations between the performance traits of Nile tilapia (Oreochromis niloticus) of the GIFT lineage during different stages of the production cycle. The Nile tilapias were grown in net cages. Daily total weight gain, live weight, and daily weight gain were measured in four periods, in approximately 30-day intervals. One-trait analyses were performed for all biometric measurements, and, in the two-trait analyses, measurements of the same traits were combined in pairs and to daily total weight gain. Estimated heritabilities ranged from 0.15 to 0.11 for live weight, from 0.16 to 0.09 for daily weight gain, and from 0.17 to 0.12 for daily total weight gain in the one-trait analyses. Estimated values of genetic correlation for live weight and daily weight gain, associated with daily total weight gain, ranged from 0.37 to 0.98 and from 0.74 to 0.88 , respectively. The high genetic correlation estimated between live weight in intermediate biometric measurements and daily total weight gain suggests that selection for growth rate may be performed earlier.
\end{abstract}

Index terms: Oreochromis niloticus, genetic correlations, heritability, GIFT strain, growth rate.

\section{Introdução}

A tilápia-do-nilo, linhagem GIFT, é conhecida pelo alto desempenho produtivo e rusticidade (Khaw et al., 2012). Um programa de melhoramento desta espécie iniciou-se em 1988, nas Filipinas (Gupta \& Acosta, 2004; Li et al., 2006), e a base populacional foi composta por quatro linhagens comerciais de tilápias da Ásia e quatro linhagens silvestres de origem africana (Bentsen et al., 1998; Gupta \&
Acosta, 2004). No Brasil, a linhagem GIFT foi introduzida em 2005, em parceria com o WorldFish Center e a Universidade Estadual de Maringá (Lupchinski Júnior et al., 2008).

Nos programas de melhoramento genético de tilápias já consolidados, a taxa de crescimento é o principal objetivo de seleção (Rutten et al., 2004), e utilizam-se informações de pedigree para a predição de valores genéticos. No entanto, em alguns trabalhos, o rendimento de filé (Rutten et al., 2005a; Gjerde et al., 
2012), a coloração externa de machos (Rajaee et al., 2010) e a tolerância ao frio (Charo-Karisa et al., 2005) foram utilizados como objetivos de seleção para tilápiado-nilo.

Em programas de melhoramento, as estimativas precisas de parâmetros genéticos são importantes para a escolha acurada dos animais geneticamente superiores que devem compor o plantel de reprodução. $\mathrm{Na}$ maioria dos trabalhos, os parâmetros genéticos são estimados para características mensuradas em idades que variam de 118 a 311 dias (Rutten et al., 2005a, 2005b; Charo-Karisa et al., 2005; Gjerde et al., 2012). Contudo, são poucos os estudos que avaliam as correlações entre as mensurações de diferentes idades (Langholz, 1990; Rutten et al., 2005b). Essas informações podem contribuir para a seleção precoce dos animais, o que diminui o intervalo entre gerações e aumenta o ganho genético anual.

O conhecimento da estrutura de associação entre diferentes características medidas ao longo do tempo pode fornecer informações importantes quanto à definição de critérios de seleção em programas de melhoramento genético de peixes.

O objetivo deste trabalho foi estimar as herdabilidades e a estrutura de correlações genéticas entre as características de desempenho de tilápia-do-nilo da linhagem GIFT, em diferentes estágios do ciclo de produção.

\section{Material e Métodos}

O conjunto de dados foi obtido a partir de progênies de tilápia [Oreochromis niloticus (Linnaeus, 1758)] da linhagem GIFT, proveniente do acasalamento de 180 animais do plantel de reprodução do programa de melhoramento genético da Universidade Estadual de Maringá (UEM), na estação reprodutiva de novembro de 2008 a fevereiro de 2009. Os reprodutores foram mantidos em hapas individuais de $1 \mathrm{~m}^{3}$ (1x1x1 m), em tanque escavado na Estação de Piscicultura da UEM, no Distrito de Floriano, Maringá, Estado do Paraná. Utilizou-se o sistema hierárquico de acasalamento de um macho com duas fêmeas. Os animais eram monitorados três vezes por semana para verificar a ocorrência de desovas ou a manifestação de sinais de desova iminente (ventre abaulado, papila urogenital avermelhada e intumescida). Os casais permaneciam juntos até a detecção da desova. Após a eclosão, as larvas permaneceram nos hapas junto das mães até o final da estação reprodutiva. Este procedimento gerou a fonte de variação, considerada no modelo, de ambiente comum de larvicultura.

Ao final da estação reprodutiva, 100 alevinos de cada uma das 58 famílias foram transferidos para a estrutura de alevinagem e mantidos em hapas de $1 \mathrm{~m}^{3}$, em tanque escavado. Estes animais foram divididos em dois grupos de 50 alevinos e distribuídos, intencionalmente, em lugares diferentes dentro do tanque, para minimizar o efeito do ambiente sob as famílias e evitar que estes fossem beneficiados com diferenças de qualidade de água, disponibilidade de oxigênio e outros fatores ambientais. Dessa forma, gerou-se o efeito de ambiente comum de alevinagem, considerado no modelo para estimação dos parâmetros genéticos.

Ao atingirem peso médio de $15 \mathrm{~g}$, os alevinos foram identificados, individualmente, por meio de microchips "passive integrated transponder tags" (PITT). Cerca de uma semana após a identificação, os animais foram transferidos para o sistema de cultivo, em tanques-rede, no Rio do Corvo, Diamante do Norte, PR, e alocados em dois tanques-rede de $6 \mathrm{~m}^{3}(2 \times 2 \times 1,5 \mathrm{~m})$, com mesma densidade e representantes de todas as famílias.

No período de cultivo em tanques-rede, de junho a outubro de 2009, foram realizadas quatro biometrias, com intervalos aproximados de 30 dias, o que correspondeu a uma biometria no outono, duas no inverno e uma na primavera. As informações anotadas foram: peso vivo, sexo e idade.

A variável ganho em peso diário foi medida de duas formas. Para caracterizar o ganho em peso diário na fase de cultivo, foi dividida a diferença dos pesos pelo intervalo de dias entre as biometrias. Já, para descrever o ganho em peso diário acumulado, foi calculada a divisão do peso em cada biometria pela idade correspondente do animal.

Assim, o ganho em peso na fase de cultivo (GPD) resultou em quatro características: GPD1, GPD2, GPD3 e GPD4, relativas ao ganho em peso diário da identificação à primeira biometria, da primeira à segunda, da segunda à terceira e da terceira à quarta biometrias, respectivamente. A variável ganho em peso diário total (GPT) também resultou em quatro características: GPT1, GPT2, GPT3 e GPT4, referentes aos ganhos em peso diários até a primeira, a segunda, a terceira e a quarta biometrias, respectivamente.

Pesq. agropec. bras., Brasília, v.48, n.8, p.816-824, ago. 2013 DOI: 10.1590/S0100-204X2013000800002 
Para a estimação dos componentes de (co)variância e dos parâmetros genéticos, utilizou-se o modelo, $\mathrm{y}=\mathrm{X} \beta+\mathrm{Z}_{1} \mathrm{a}+\mathrm{Z}_{2} \mathrm{~m}+\mathrm{Z}_{3} \mathrm{c}+\varepsilon$, em que: y é o vetor de observações das características analisadas; $\beta$ é o vetor dos efeitos ambientais identificáveis; e a, m, c e $\varepsilon$ são os vetores dos efeitos genéticos aditivos diretos, do efeito de ambiente comum de larvicultura, do efeito de ambiente comum de alevinagem e dos erros aleatórios, respectivamente. As matrizes $\mathrm{X}$, $Z_{1}, Z_{2}$ e $Z_{3}$ são referentes às incidências dos efeitos ambientais identificáveis, genéticos aditivos diretos, de ambiente comum de larvicultura e de ambiente comum de alevinagem, respectivamente. Ao admitir-se que $\mathrm{a}, \mathrm{m}, \mathrm{c}$ e e apresentam distribuição conjunta normal multivariada, tem-se:

$$
\begin{gathered}
{\left[\begin{array}{c}
\mathrm{a} \\
\mathrm{m} \\
\mathrm{c} \\
\mathrm{e}
\end{array}\right] \sim \operatorname{NMV}\left\{\left[\begin{array}{l}
\phi \\
\phi \\
\phi \\
\phi
\end{array}\right],\left[\begin{array}{cccc}
\mathrm{A} \sigma_{\mathrm{a}}^{2} & \phi & \phi & \phi \\
\phi & \mathrm{I}_{\mathrm{h}} \sigma_{\mathrm{m}}^{2} & \phi & \phi \\
\phi & \phi & \mathrm{I}_{\mathrm{c}} \sigma_{\mathrm{c}}^{2} & \phi \\
\phi & \phi & \phi & \mathrm{I} \sigma_{\mathrm{e}}^{2}
\end{array}\right]\right\} ;} \\
\operatorname{Var}(\mathrm{y})=\mathrm{Z}_{1} \mathrm{AZ} \mathrm{Z}_{1}^{\prime} \sigma_{\mathrm{a}}^{2}+\mathrm{Z}_{2} \mathrm{Z}_{2}^{\prime} \sigma_{\mathrm{m}}^{2}+\mathrm{Z}_{3} \mathrm{Z}_{3}^{\prime} \mathrm{s}+\mathrm{I}_{\mathrm{n}} \sigma_{\mathrm{e}}^{2},
\end{gathered}
$$

em que: A é a matriz de parentesco entre os animais; $\sigma_{\mathrm{a}}^{2}$ é a variância genética aditiva direta; $\sigma_{\mathrm{m}}^{2}, \sigma_{\mathrm{c}}^{2}$ e $\sigma_{\mathrm{e}}^{2}$ são as variâncias do efeito de ambiente comum de larvicultura, de ambiente comum de alevinagem e residual, respectivamente; $I_{h}$ é a matriz identidade de ordem $\mathrm{h}$, sendo $\mathrm{h}$ igual ao número de famílias de irmãos completos; $I_{c}$ é a matriz identidade de ordem c, sendo c igual ao número de hapas de alevinagem; e $\mathrm{I}_{\mathrm{n}}$ é a matriz identidade, de ordem $\mathrm{n}$, sendo $\mathrm{n}$ igual ao número de observações.

Para as análises bicaracter, têm-se as equações: $\mathrm{G}=\mathrm{A} \otimes \mathrm{G}_{0}$, em que $\mathrm{G}_{0}$ é a matriz de variâncias genéticas das características; $\mathrm{P}=\mathrm{I}_{\mathrm{h}} \otimes \mathrm{P}_{0}$, em que $\mathrm{P}_{0}$ é a matriz de variâncias relativas ao efeito de ambiente comum de larvicultura; $C=\mathrm{I}_{c} \otimes \mathrm{C}_{0}$, em que $\mathrm{C}_{0}$ é a matriz de variâncias relativas ao efeito de ambiente comum de alevinagem; e $R=I_{n} \otimes R_{0}$, em que $R_{0}$ é a matriz de variância e covariâncias residuais. As matrizes $\mathrm{G}_{0}, \mathrm{P}_{0}$, $\mathrm{C}_{0}$ e $\mathrm{R}_{0}$ têm ordem 2 nas análises bicaracter.

As análises unicaracter foram realizadas para peso vivo, GPD e GPT com informações de todas as biometrias. Para as análises bicaracter, a estratégia foi combinar, duas a duas, as medidas da mesma característica de diferentes biometrias, que foram consideradas como características distintas. Além disso, foram combinadas as informações de GPT4 com as de peso vivo e de GPD de todas as biometrias.

Os componentes de (co)variância e os parâmetros genéticos foram estimados por inferência bayesiana, por meio do programa computacional "Multiple trait Gibbs sampler for animal models" (MTGSAM) (Van Tassel \& Van Vleck, 1995). Consideraram-se os efeitos genéticos aditivos, de ambiente comum de larvicultura, de ambiente comum de alevinagem e residual como tendo distribuição a priori normal para as análises unicaracter e bicaracter. Para os demais efeitos, considerou-se a distribuição a priori como plana ("flat prior"). Para os componentes de (co)variância, considerou-se a distribuição a priori qui-quadrado invertida e wishard invertida para as análises unicaracter e bicaracter, respectivamente.

As distribuições posteriores foram obtidas por meio, inicialmente, de 500.000 ciclos, tendo-se retirado amostras a cada dez ciclos, após a eliminação dos 50.000 ciclos iniciais, o que totalizou 45.000 amostras dos componentes de (co)variância. A partir destas amostras, foi estimada a média a posteriori e os intervalos de credibilidade de $95 \%$ para os componentes de (co)variância e os demais parâmetros genéticos.

Para avaliar a convergência das cadeias geradas, utilizou-se o teste de Heidelberger \& Welch, descrito por Cowles et al. (1995), disponível na biblioteca "Convergence diagnosis and output analysis" (CODA), implementada no programa $\mathrm{R}$, versão 2.12.0.

Foram estimadas as correlações de Pearson e Spearman entre os valores genéticos preditos para as características de peso vivo, GPD e GPT entre as diferentes biometrias. Adicionalmente, estimou-se as correlações entre GPT4 e os pesos vivos e GPD nas diferentes biometrias.

A partir dos valores genéticos preditos para cada característica, foram selecionados um macho e duas fêmeas de cada família. Este processo foi realizado para todas as características (peso vivo, GPD e GPT) em cada biometria. A partir daí verificou-se o percentual de indivíduos selecionados comuns para a mesma característica nas diferentes biometrias. A classificação de GPT4 foi comparada com as classificações de peso vivo e GPD em todas as biometrias. Foi calculado, adicionalmente, a percentagem de coincidência das 29 melhores famílias, utilizou-se a médias dos valores genético dos membros das famílias com critério de classificação. 


\section{Resultados e Discussão}

Os valores de herdabilidade estimados para as características peso vivo, GPD e GPT, ao longo do período de cultivo, foram de média magnitude, com valores oscilando de 0,154 a 0,112 , de 0,165 a 0,098 e de 0,169 a 0,117 , respectivamente, para as análises unicaracter (Tabela 1). A utilização de informações adicionais, com a combinação das características em análises bicaracter, não promoveu mudanças nas estimativas, pois estas estão contidas nas estimativas intervalares obtidas nas análises unicaracter.

As estimativas pontuais das herdabilidades para peso vivo, GPD e GPT, em modelos uni e bicaracter, apresentaram comportamento decrescente até a terceira biometria, com acréscimo na última biometria, o que indica redução da participação relativa das diferenças genéticas aditivas na variação total. Rutten et al. (2005b), Rezk et al. (2009) e Santos et al. (2011) estimaram herdabilidades que variaram de 0,10 a 0,25 , para peso vivo à identificação e à despesca de tilápia-do-nilo, respectivamente.

Em trabalho com truta arco-íris (Oncorhynchus mykiss), Kause et al. (2003) verificaram redução dos valores de herdabilidade para fêmeas à medida que estas se tornaram sexualmente maduras. Já Dupont-Nivet et al. (2010), ao estudar a mesma espécie, observaram menor valor de herdabilidade quando utilizados machos imaturos e maduros sexualmente no conjunto de dados. Portanto, a redução da herdabilidade, no presente trabalho, pode ser indicativo da maturidade dos animais, o que ocasiona mudanças fisiológicas e, consequentemente, aumento de efeitos não identificáveis.

Nas análises unicaracter para os efeitos ambientais comuns, constatou-se que o ambiente comum de larvicultura $\left(\mathrm{C}^{2}\right)$ permaneceu constante para peso vivo, GPT e GPD, o que indica que a ausência da incubação artificial, o prolongado período que os animais permaneceram juntos no hapa da mãe, a maior densidade de animais e a maior competição por alimento, acarretaram a maior participação relativa da variação resultante do efeito, o qual, se for desconsiderado no modelo, poderia comprometer a acurácia da avaliação genética.

$\mathrm{O}$ efeito de ambiente comum de alevinagem $\left(\mathrm{W}^{2}\right)$, para as características peso vivo, GPD e GPT, nas análises unicaracter, apresentou redução de valores com o aumento da idade dos animais, o que indica que a alocação dos animais nos hapas de alevinagem, em diferentes locais dentro do tanque, pode minimizar os efeitos ambientais e contribuir para a redução da participação relativa na variação total das características medidas.

$\mathrm{Na}$ técnica de incubação artificial, somente um valor é estimado para o efeito ambiental comum, referente à permanência dos irmãos em um mesmo hapa até a identificação. Ao utilizar essa técnica,

Tabela 1. Valores de herdabilidade $\left(\mathrm{h}^{2}\right)$, ambiente comum de larvicultura $\left(\mathrm{C}^{2}\right)$, ambiente comum de alevinagem $\left(\mathrm{W}^{2}\right)$ e variância fenotípica $\left(\sigma^{2}\right)$ das análises unicaracter para peso vivo, ganho em peso diário e ganho em peso diário total, em todas as biometrias.

\begin{tabular}{|c|c|c|c|c|c|c|c|c|}
\hline \multirow[t]{2}{*}{ Biometria } & \multicolumn{2}{|c|}{$\mathrm{h}^{2}$} & \multicolumn{2}{|c|}{$\mathrm{C}^{2}$} & \multicolumn{2}{|c|}{$\mathrm{W}^{2}$} & \multicolumn{2}{|c|}{$\sigma^{2}$} \\
\hline & Intervalo & Média & Intervalo & Média & Intervalo & Média & Intervalo & Média \\
\hline & \multicolumn{8}{|c|}{ Peso vivo } \\
\hline 1 & $0,04-0,31$ & 0,154 & $0,01-0,13$ & 0,054 & $0,01-0,08$ & 0,040 & $452-541$ & 493,15 \\
\hline 2 & $0,04-0,29$ & 0,137 & $0,02-0,12$ & 0,059 & $0,01-0,06$ & 0,029 & $1.105-1.315$ & $1.200,98$ \\
\hline 3 & $0,04-0,23$ & 0,112 & $0,02-0,11$ & 0,057 & $0,01-0,05$ & 0,025 & $2.511-2.959$ & $2.718,41$ \\
\hline \multirow[t]{2}{*}{4} & $0,04-0,25$ & 0,118 & $0,02-0,12$ & 0,060 & $0,01-0,05$ & 0,024 & $4.687-5.532$ & $5.074,41$ \\
\hline & \multicolumn{8}{|c|}{ Ganho em peso diário } \\
\hline 1 & $0,04-0,35$ & 0,165 & $0,02-0,15$ & 0,069 & $0,01-0,08$ & 0,038 & $0,09-0,11$ & 0,099 \\
\hline 2 & $0,03-0,21$ & 0,101 & $0,02-0,10$ & 0,051 & $0,01-0,04$ & 0,021 & $0,13-0,16$ & 0,143 \\
\hline 3 & $0,03-0,21$ & 0,098 & $0,02-0,10$ & 0,048 & $0,01-0,04$ & 0,020 & $0,20-0,24$ & 0,219 \\
\hline \multirow[t]{2}{*}{4} & $0,04-0,26$ & 0,129 & $0,01-0,10$ & 0,048 & $0,008-0,04$ & 0,019 & $0,72-0,85$ & 0,781 \\
\hline & \multicolumn{8}{|c|}{ Ganho em peso diário total } \\
\hline 1 & $0,04-0,33$ & 0,169 & $0,01-0,13$ & 0,052 & $0,02-0,09$ & 0,044 & $0,01-0,02$ & 0,017 \\
\hline 2 & $0,04-0,30$ & 0,149 & $0,01-0,13$ & 0,057 & $0,01-0,06$ & 0,029 & $0,02-0,03$ & 0,027 \\
\hline 3 & $0,04-0,25$ & 0,117 & $0,02-0,12$ & 0,056 & $0,01-0,05$ & 0,024 & $0,04-0,05$ & 0,041 \\
\hline 4 & $0,06-0,27$ & 0,127 & $0,02-0,12$ & 0,059 & $0,01-0,05$ & 0,023 & $0,06-0,07$ & 0,062 \\
\hline
\end{tabular}


Charo-Karisa et al. (2007), Bentsen et al. (2012) e Gjerde et al. (2012) encontraram estimativas do efeito de ambiente comum materno que variaram de 0,05 a 0,23 , para peso corporal, em tilápia-do-nilo.

A participação da variância de ambiente comum materno para peso aos 180 dias, estimada por Nguyen et al. (2010), foi de cerca de $8 \%$ na variação total, valor muito próximo ao observado no presente trabalho, quando utilizadas as estimativas conjuntas de $\mathrm{C}^{2}$ e $\mathrm{W}^{2}$, em todas as biometrias. Ao considerarem 120 dias de cultivo, Nguyen et al. (2007) obtiveram valores de 0,18 para o mesmo efeito, o que mostra que houve redução da importância relativa do efeito comum de família na variação total com o aumento da idade.

$\mathrm{Na}$ estimativa das associações existentes entre as medidas das características de desempenho ao longo do período de cultivo, constatou-se que quanto mais próximas as biometrias, maior a associação genética e fenotípica. Assim, valores de correlações genéticas e fenotípicas foram superiores a 0,90 entre as biometrias 1 e 2,2 e 3 e 3 e 4, para peso vivo e GPT. Para GPD, entre as biometrias mais próximas, os valores das correlações fenotípicas foram inferiores a 0,70, enquanto, para correlações genéticas, as estimativas foram superiores a 0,70 e inferiores a 0,80 (Tabela 2).

As estimações das correlações genéticas entre GPT4 e peso vivo ao longo do período de cultivo foram crescentes, com valores superiores a 0,90 para as associações nas biometrias 3 e 4 . Nas correlações entre GPT4 e GPD, observou-se maior associação com as biometrias intermediárias, coincidentes com o período em que a temperatura é mais baixa, entre julho e setembro, quando ocorre o inverno na região em que os animais foram cultivados (Tabela 2).

Ao se considerar que a seleção dos animais, para compor o próximo plantel de reprodução, é realizada quando os animais atingem cerca de cinco meses de cultivo, a predição dos valores genéticos poderia ser adiantada com o uso de dados medidos precocemente. A utilização das informações de peso vivo medido com um ou dois meses de antecedência pode resultar em resposta à seleção indireta para velocidade de crescimento, pois houve forte associação genética com o GPT4, com correlações superiores a 0,812 e 0,949, respectivamente. Esses resultados são indicativos de que as informações coletadas na terceira biometria poderiam ser utilizadas como critérios de seleção alternativos.

Resultados semelhantes foram obtidos por Rutten et al. (2005b), ao utilizar regressão aleatória, os quais verificaram redução da associação genética entre pesos à medida que aumentava o intervalo de tempo entre as biometrias. Os valores de correlações genéticas do peso vivo (P1) no início e no final do período de cultivo (P4), estimados no presente trabalho, são próximos aos valores de 0,233 e 0,40 encontrados por Rezk et al. (2009) e Santos et al. (2011), respectivamente.

As correlações de Spearman e Pearson entre peso vivo, GPD e GPT, nas diferentes biometrias, foram utilizadas como indicadores do impacto das características GPD e peso vivo como critério de seleção. Os valores de correlação de Pearson foram superiores a 0,80 , e os valores de correlação de Spearman oscilaram entre 0,769 e 0,967, para peso vivo, e entre 0,775 e 0,967 para GPT. Para GPD, verificou-se correlação mais fraca dos valores genéticos e de ranking entre as diferentes biometrias, quando comparado com peso vivo e GPT, com valores máximos de 0,77 e 0,81 para as correlações de Pearson

Tabela 2. Média das herdabilidades (diagonal principal), correlações fenotípicas (acima da diagonal) e correlações genéticas (abaixo da diagonal) das análises bicaracter para peso vivo, ganho em peso diário e ganho em peso diário total, em todas as biometrias, e para ganho em peso diário total na quarta biometria (GPT4), com peso vivo e ganho em peso diário.

\begin{tabular}{|c|c|c|c|c|c|}
\hline Biometria & 1 & 2 & 3 & 4 & GPT4 \\
\hline & \multicolumn{5}{|c|}{ Peso vivo } \\
\hline 1 & 0,176 & 0,947 & 0,886 & 0,820 & 0,594 \\
\hline 2 & 0,940 & 0,146 & 0,953 & 0,899 & 0,889 \\
\hline 3 & 0,838 & 0,912 & 0,115 & 0,957 & 0,952 \\
\hline 4 & 0,733 & 0,820 & 0,920 & 0,123 & 0,993 \\
\hline \multirow[t]{2}{*}{ GPT4 } & 0,397 & 0,812 & 0,949 & 0,980 & 0,132 \\
\hline & \multicolumn{5}{|c|}{ Ganho em peso diário } \\
\hline 1 & 0,174 & 0,690 & 0,635 & 0,496 & 0,808 \\
\hline 2 & 0,756 & 0,096 & 0,699 & 0,587 & 0,846 \\
\hline 3 & 0,384 & 0,703 & 0,091 & 0,635 & 0,785 \\
\hline 4 & 0,483 & 0,699 & 0,792 & 0,147 & 0,828 \\
\hline \multirow[t]{2}{*}{ GPT4 } & 0,736 & 0,878 & 0,865 & 0,848 & 0,134 \\
\hline & \multicolumn{5}{|c|}{ Ganho em peso diário total } \\
\hline 1 & 0,196 & 0,948 & 0,887 & 0,821 & - \\
\hline 2 & 0,951 & 0,170 & 0,952 & 0,898 & - \\
\hline 3 & 0,855 & 0,916 & 0,128 & 0,955 & - \\
\hline 4 & 0,767 & 0,843 & 0,927 & 0,135 & - \\
\hline
\end{tabular}


e Spearman, respectivamente (Tabela 3). Constatouse que, para as três características, as informações coletadas em biometrias mais próximas implicaram em maiores valores das correlações, o que está de acordo com os resultados encontrados para as correlações genéticas.

Na seleção do melhor macho e das duas melhores fêmeas por família, verificou-se que, para peso vivo e GPT, em machos, e, para GPD, em ambos os sexos, a maior percentagem de animais selecionados comuns foi observada entre a biometria 1 e 2, com valores de 47,46 a $77,97 \%$, para machos, e de $62,39 \%$ para fêmeas. No entanto, para peso vivo e GPT, a maior percentagem de coincidências para as fêmeas foi encontrada entre as biometrias 3 e 4 (Tabela 4).

Ao se estimar a correlação de Spearman de todas as famílias entre as biometrias, para o critério de seleção, os valores superiores a 0,93 entre biometrias mais próximas indicaram pequena modificação na classificação das famílias para a característica GPT (Tabela 5). Na seleção das 29 melhores famílias, observaram-se altas coincidências das classificações para peso vivo e GPT, com valores entre 75,86 e $93,10 \%$, entre as diferentes biometrias (Tabelas 5 e 6). Contudo, para GPD, o percentual de coincidência

Tabela 3. Correlação de Pearson (acima da diagonal) e correlação de Spearman (abaixo da diagonal) para as características peso vivo, ganho em peso diário e ganho em peso diário total, em todas as biometrias, e entre ganho em peso diário total na quarta biometria (GPT4), com peso vivo e ganho em peso diário.

\begin{tabular}{|c|c|c|c|c|c|}
\hline Biometria & 1 & 2 & 3 & 4 & GPT4 \\
\hline & \multicolumn{5}{|c|}{ Peso vivo } \\
\hline 1 & - & 0,969 & 0,891 & 0,805 & 0,797 \\
\hline 2 & 0,960 & - & 0,956 & 0,891 & 0,885 \\
\hline 3 & 0,870 & 0,945 & - & 0,966 & 0,957 \\
\hline 4 & 0,769 & 0,869 & 0,967 & - & 0,995 \\
\hline \multirow[t]{2}{*}{ GPT4 } & 0,766 & 0,868 & 0,964 & 0,998 & - \\
\hline & \multicolumn{5}{|c|}{ Ganho em peso diário } \\
\hline 1 & - & 0,770 & 0,604 & 0,515 & 0,818 \\
\hline 2 & 0,759 & - & 0,770 & 0,682 & 0,913 \\
\hline 3 & 0,620 & 0,755 & - & 0,769 & 0,877 \\
\hline 4 & 0,484 & 0,691 & 0,810 & - & 0,859 \\
\hline \multirow[t]{2}{*}{ GPT4 } & 0,794 & 0,907 & 0,903 & 0,859 & - \\
\hline & \multicolumn{5}{|c|}{ Ganho em peso diário total } \\
\hline 1 & - & 0,970 & 0,895 & 0,814 & - \\
\hline 2 & 0,962 & - & 0,956 & 0,894 & - \\
\hline 3 & 0,872 & 0,943 & - & 0,967 & - \\
\hline 4 & 0,775 & 0,869 & 0,967 & - & - \\
\hline
\end{tabular}

de famílias selecionadas foi inferior, com valores que oscilaram entre 62,07 e $75,86 \%$, entre as diferentes biometrias. As maiores coincidências de famílias selecionadas para peso vivo e GPT foram observadas nas biometrias mais próximas; contudo, para GPD, este comportamento não ficou evidenciado (Tabela 6).

As correlações de Pearson e Spearman estimadas entre GPT4 e peso vivo e entre GPT4 e GPD, nas diferentes biometrias, apontaram forte associação com valores superiores a 0,75 . A comparação das classificações de GPT4 com peso vivo e de GPT4 com GPD, nas diferentes biometrias, indicou maior coincidência com a biometria 4 e 2 , respectivamente (Tabela 3).

Para GPT4, associado ao peso vivo, em ambos os sexos, as percentagens de animais comuns foram crescentes ao longo do período de cultivo, tendo alcançado valores de 99,15 e 100\% para fêmeas e machos, respectivamente, na última biometria (Tabela 4). Em relação ao GPT4 com GPD, o percentual de machos selecionados comuns não excedeu a $60 \%$, e, para fêmeas, este valor foi menor que 75\% (Tabela 4).

Padala-de Vera \& Eknath (1993) encontraram valores de correlação de Pearson baixos e negativos ao comparar o peso corporal de tilápia-do-nilo em

Tabela 4. Percentagem de coincidência de machos (acima da diagonal) e fêmeas (abaixo da diagonal) selecionados para as características peso vivo, ganho em peso diário e ganho em peso diário total, em todas as biometrias, e entre ganho em peso diário total na quarta biometria (GPT4), com peso vivo e ganho em peso diário.

\begin{tabular}{|c|c|c|c|c|c|}
\hline Biometria & 1 & 2 & 3 & 4 & GPT4 \\
\hline & \multicolumn{5}{|c|}{ Peso vivo $(\%)$} \\
\hline 1 & - & 77,97 & 67,80 & 57,63 & 57,63 \\
\hline 2 & 75,21 & - & 72,88 & 62,71 & 62,71 \\
\hline 3 & 64,10 & 84,62 & - & 74,58 & 74,58 \\
\hline 4 & 56,41 & 69,23 & 79,49 & - & 100,00 \\
\hline \multirow[t]{2}{*}{ GPT4 } & 57,26 & 70,09 & 79,49 & 99,15 & - \\
\hline & \multicolumn{5}{|c|}{ Ganho em peso diário (\%) } \\
\hline 1 & - & 47,46 & 27,12 & 28,81 & 54,24 \\
\hline 2 & 62,39 & - & 33,90 & 35,59 & 55,93 \\
\hline 3 & 55,56 & 55,56 & - & 32,20 & 47,46 \\
\hline 4 & 50,43 & 57,26 & 55,56 & - & 57,63 \\
\hline \multirow[t]{2}{*}{ GPT4 } & 63,25 & 71,79 & 72,65 & 73,50 & - \\
\hline & \multicolumn{5}{|c|}{ Ganho em peso diário total (\%) } \\
\hline 1 & - & 77,97 & 67,80 & 57,63 & - \\
\hline 2 & 76,92 & - & 72,88 & 62,71 & - \\
\hline 3 & 66,67 & 85,47 & - & 74,58 & - \\
\hline 4 & 57,26 & 70,09 & 80,34 & - & - \\
\hline
\end{tabular}

Pesq. agropec. bras., Brasília, v.48, n.8, p.816-824, ago. 2013 DOI: 10.1590/S0100-204X2013000800002 
fase inicial de cultivo (108 dias) com o peso em fases sucessivas até 186 dias, o que evidencia que o desempenho na fase inicial de crescimento é mau indicador do desempenho em fases mais tardias.

Ao trabalhar com modelo de regressão aleatória, Rutten et al. (2005b) verificaram que a seleção para peso corporal teria máxima resposta quando baseada na idade aproximada de 260 dias. Entretanto, quando a resposta da seleção é expressa como resposta ao ano, a idade ideal de seleção diminui ainda mais, próximo aos 175 dias, em razão de um reduzido intervalo de gerações. Esses resultados corroboram os do presente trabalho, ao se considerar que a segunda biometria abrange animais com idade média de 211 dias.

Os resultados obtidos no presente trabalho são indicativos de que a seleção para ganho em peso diário total durante o período de cultivo pode conduzir a respostas indiretas para peso vivo, principalmente nas biometrias 3 e 4 . Isso aponta que o processo de seleção pode ser realizado precocemente, pois os valores de correlação de Spearman, de Pearson, percentagem de coincidência para machos, fêmeas e famílias selecionadas para peso vivo e GPT, nos diferentes períodos de cultivo, sugerem que a maioria dos animais selecionados precocemente estaria no grupo de animais selecionados por meio do critério de ganho em peso diário total na última biometria.

Contudo, para ganho em peso diário, os resultados são indicativos de que o desempenho nos diferentes períodos de cultivo são independentes do desempenho no período total, o que pode ser atribuído à existência de genótipos que apresentaram diferenças no desempenho em períodos distintos.

Ao se compararem as classificações entre os períodos estudados, observaram-se diferenças no ranqueamento de machos, fêmeas e famílias, para peso vivo, o que é evidenciado pelo distanciamento das medidas. Para GPD, estas diferenças são causadas por

Tabela 5. Percentagem de coincidência das 29 melhores famílias (acima da diagonal) e correlações de Spearman de todas as famílias (abaixo da diagonal) para ganho em peso diário total, em todas as biometrias.

\begin{tabular}{lcccc}
\hline Biometria & 1 & 2 & 3 & 4 \\
\hline 1 & - & 89,65 & 79,31 & 75,86 \\
2 & 0,954 & - & 86,21 & 79,31 \\
3 & 0,843 & 0,934 & - & 93,10 \\
4 & 0,719 & 0,845 & 0,958 & - \\
\hline
\end{tabular}

circunstâncias específicas das fases, provavelmente relacionadas às condições ambientais, o que sugere a ocorrência de interação genótipo $\mathrm{x}$ ambiente, que pode ter se manifestado na forma de alteração de magnitude dos valores genéticos (Tabelas 3 e 4) ou de alterações no ranking (Tabela 6).

Em estudo para quantificar o efeito do genótipo e do ambiente em juvenis de tilápia-do-nilo cultivadas em hapas dentro de dois tanques escavados, Charo-Karisa et al. (2006) identificaram correlação genética de -0,27 entre peso corporal de alevinos, o que evidencia a interação genótipo $\mathrm{x}$ ambiente, possivelmente ocasionada pelas diferenças da quantidade de oxigênio dissolvido na água, em ambos os tanques de cultivo.

Ao estimarem as correlações genéticas e fenotípicas para compor uma população de tilápia-do-nilo selecionadas para reprodução, Eknath et al. (2007) constataram que são altas as correlações genéticas entre o mesmo ambiente de cultivo. Porém, quando associados ambientes diferentes, como o cultivo em tanque-rede e tanque escavado, a correlação genética pode ser menor e mais variável, o que indica que pode haver interação genótipo $\mathrm{x}$ ambiente em razão das diferenças de cultivo (Bentsen et al., 2012; Khaw et al., 2012).

Assim, a existência de diferentes grupos de animais selecionados em função dos períodos de cultivo indica que o ganho em peso diário pode sofrer influência de conjuntos diferentes de genes e que a alteração na classificação implica na seleção de tilápias que podem ser ineficientes, do ponto de vista produtivo, para determinado ambiente de cultivo, o que ressalta a necessidade de diferentes programas de seleção se o critério utilizado for ganho em peso diário, medido em fases distintas.

Tabela 6. Percentagem de coincidência das 29 melhores famílias selecionadas a partir das informações de peso vivo (acima da diagonal) e ganho em peso diário (abaixo da diagonal), nas diferentes biometrias, e entre ganho em peso diário total na quarta biometria (GPT4).

\begin{tabular}{lccccc}
\hline Biometria & 1 & 2 & 3 & 4 & GPT4 \\
\hline 1 & - & 93,10 & 82,76 & 75,86 & 75,86 \\
2 & 75,86 & - & 86,21 & 79,31 & 79,31 \\
3 & 62,07 & 75,86 & - & 93,10 & 93,10 \\
4 & 68,97 & 72,41 & 75,86 & - & 100,00 \\
GPT4 & 75,86 & 82,76 & 86,21 & 82,76 & - \\
\hline
\end{tabular}




\section{Conclusões}

1. Os valores de herdabilidades indicam a existência de resposta à seleção para as características de desempenho de tilápia-do-nilo.

2. Há forte associação genética e fenotípica entre as medidas da mesma característica, com redução dos intervalos entre as biometrias.

3. O critério de seleção ganho em peso total apresenta forte associação genética com peso vivo e ganho em peso diário.

4. A seleção pode ser feita precocemente, em razão da forte associação genética entre ganho em peso diário total e peso vivo, medido ao longo do período de cultivo.

\section{Referências}

BENTSEN, H.B.; EKNATH, A.E.; PALADA-DE VERA, M.S.; DANTING, J.C.; BOLIVAR, H.L.; REYES, R.A.; DIONISIO, E.E.; LONGALONG, F.M.; CIRCA, A.V.; TAYAMEN, M.M.; GJERDE, B. Genetic improvement of farmed tilapias: growth performance in a complete diallel cross experiment with eight strains of Oreochromis niloticus. Aquaculture, v.160, p.145-173, 1998. DOI: 10.1016/S0044-8486(97)00230-5.

BENTSEN, H.B.; GJERDE, B.; NGUYEN, N.H.; RYE, M.; PONZONI, R.W.; VERA, M.S.P. de; BOLIVAR, H.L.; VELASCO, R.R.; DANTING, J.C.; DIONISIO, E.E.; LONGALONG, F.M.; REYES, R.A.; ABELLA, T.A.; TAYAMEN, M.M.; EKNATH, A.E. Genetic improvement of farmed tilapias: genetic parameters for body weight at harvest in Nile tilapia (Oreochromis niloticus) during five generations of testing in multiple environments. Aquaculture, v.338-341, p.56-65, 2012. DOI: 10.1016/j. aquaculture.2012.01.027.

CHARO-KARISA,H.;BOVENHUIS,H.;REZK,M.A.;PONZONI, R.W.; ARENDONK, J.A.M. van; KOMEN, H. Phenotypic and genetic parameters for body measurements, reproductive traits and gut length of Nile tilapia (Oreochromis niloticus) selected for growth in low-input earthen ponds. Aquaculture, v.273, p.15-23, 2007. DOI: 10.1016/j.aquaculture.2007.09.011.

CHARO-KARISA, H.; KOMEN, H.; REZK, M.A.; PONZONI, R.W.; VAN ARENDONK, J.A.M.; BOVENHUIS, H. Heritability estimates and response to selection for growth of Nile tilapia (Oreochromis niloticus) in low-input earthen ponds. Aquaculture, v.261, p.479-486, 2006. DOI: 10.1016/j.aquaculture.2006.07.007.

CHARO-KARISA, H.; REZK, M.A.; BOVENHUIS, H.; KOMEN, H. Heritability of cold tolerance in Nile tilapia, Oreochromis niloticus, juveniles. Aquaculture, v.249, p.115-123, 2005. DOI: 10.1016/j.aquaculture.2005.04.029.

COWLES, M.K.; BEST, N.G.; VINES, K. CODA: convergence diagnostics and output analysis software for Gibbs sampler output. Version 0.30. Robinson Way: Institute of Public Health, 1995.
DUPONT-NIVET, M.; CHEVASSUS, B.; MAUGER, S.; HAFRAY, P.; VANDEPUTTE, M. Side effects of sexual maturation on heritability estimates in rainbow trout (Oncorhynchus mykiss). Aquaculture Research, v.41, p.e878-e880, 2010. DOI: 10.1111/j. 1365-2109.2009.02448.x.

EKNATH, A.E.; BENTSEN, H.B.; PONZONI, R.W.; RYE, M.; NGUYEN, N.H.; THODESEN, J.; GJERDE, B. Genetic improvement of farmed tilapias: composition and genetic parameters of a synthetic base population of Oreochromis niloticus for selective breeding. Aquaculture, v.273, p.1-14, 2007. DOI: 10.1016/j.aquaculture.2007.09.015.

GJERDE, B.; MENGISTU, S.B.; ØDEGÅRD, J.; JOHANSEN, H.; ALTAMIRANO, D.S. Quantitative genetics of body weight, fillet weight and fillet yield in Nile tilapia (Oreochromis niloticus). Aquaculture, v.342-343, p.117-124, 2012. DOI: 10.1016/j. aquaculture.2012.02.015.

GUPTA, M.V.; ACOSTA, B.O. From drawing board to dining table: the success story of the GIFT project. Naga, WorldFish Center Quarterly, v.27, p.4-14, 2004.

KAUSE, A.; RITOLA, O.; PAANANEN, T.; MANTYSAARI, E.; ESKELINEN, U. Selection against early maturity in large rainbow trout Oncorhynchus mykiss: the quantitative genetics of sexual dimorphism and genotype-by-environment interactions. Aquaculture, v.228, p.53-68, 2003. DOI: 10.1016/ S0044-8486(03)00244-8.

KHAW, H.L; PONZONI, R.W.; HAMZAH, A.; ABU-BAKAR, K.R.; BIJMA, P. Genotype by production environment interaction in the GIFT strain of Nile tilapia (Oreochromis niloticus). Aquaculture, v.326, p.53-60, 2012. DOI: 10.1016/j. aquaculture.2011.11.016.

LANGHOLZ, H.J. Intraspecific variation in tilapia. In: SATUS SEMINAR, 3., 1987, Tiberias. Proceedings. Tiberias: European Aquaculture Society, 1990. p.103-118. (European Aquaculture Society. Special publication, 11).

LI, S.F.; HE, X.J.; HU, G.C.; CAI, W.Q.; DENG, X.W.; ZHOU, P.Y. Improving growth performance and caudal fin stripe pattern in selected F6-F8 generations of GIFT Nile tilapia (Oreochromis niloticus L.) using mass selection. Aquaculture Research, v.37, p.1165-1171, 2006. DOI: 10.1111/j.1365-2109.2006.01543.x.

LUPCHINSKI JÚNIOR, E.; VARGAS, L.; POVH, J.A.; RIBEIRO, R.P.; MANGOLIM, C.A.; LOPERA BARRERO, N.M. Avaliação da variabilidade das gerações G0 e F1 da linhagem GIFT de tilápiado-nilo (Oreochromis niloticus) por RAPD. Acta Scientiarum. Animal Science, v.30, p.233-240, 2008.

NGUYEN, N.H.; KHAW, H.L.; PONZONI, R.W.; HAMZAH, A.; KAMARUZZAMAN, N. Can sexual dimorphism and body shape be altered in Nile tilapia by genetic means? Aquaculture, v.272, p.S38-S46, 2007. DOI: 10.1016/j.aquaculture.2007.08.013.

NGUYEN, N.H.; PONZONI, R.W.; ABU-BAKAR, K.R.; HAMZAH, A.; KHAW, H.L.; YEE, H.Y. Correlated response in fillet weight and yield to selection for increased harvest weight in genetically improved farmed tilapia (GIFT strain), Oreochromis niloticus. Aquaculture, v.305, p.1-5, 2010. DOI: 10.1016/j. aquaculture.2010.04.007.

Pesq. agropec. bras., Brasília, v.48, n.8, p.816-824, ago. 2013 DOI: 10.1590/S0100-204X2013000800002 
PALADA-DE VERA, M.S.; EKNATH, A.E. Predictability of individual growth-rates in tilapia. Aquaculture, v.111, p.147-158, 1993. DOI: 10.1016/0044-8486(93)90033-U.

RAJAEE, A.H.; HUNTINGFORD, F.A.; RANSON, K.J.; MCANDREW, B.J.; PENMAN, D.J. The effect of male colouration on reproductive success in Nile tilapia (Oreochromis niloticus). Aquaculture, v.308, p.S119-S123, 2010. DOI: 10.1016/j. aquaculture.2010.05.039.

REZK, M.A.; PONZONI, R.W.; KHAW, H.L.; KAMEL, E.; DAWOOD, T.; JOHN, G. Selective breeding for increased body weight in a synthetic breed of Egyptian Nile tilapia, Oreochromis niloticus: response to selection and genetic parameters. Aquaculture, v.293, p.187-194, 2009. DOI: 10.1016/j. aquaculture.2009.03.019.

RUTTEN, M.J.M.; BOVENHUI, H.; KOMEN, H. Modeling fillet traits based on body measurements in three Nile tilapia strains (Oreochromis niloticus L.). Aquaculture, v.231, p.113-122, 2004. DOI: 10.1016/j.aquaculture.2003.11.002.
RUTTEN, M.J.M.; BOVENHUIS, H.; KOMEN, H. Genetic parameters for fillet traits and body measurements in Nile tilapia (Oreochromis niloticus L.). Aquaculture, v.246, p.125-132, 2005a. DOI: 10.1016/j.aquaculture.2005.01.006.

RUTTEN, M.J.M.; KOMEN, H.; BOVENHUIS, H. Longitudinal genetic analysis of Nile tilapia (Oreochromis niloticus L.) body weight using a random regression model. Aquaculture, v.246, p.101-113, 2005b. DOI: 10.1016/j.aquaculture.2004.12.020.

SANTOS, A.I.; RIBEIRO, R.P.; VARGAS, L.; MORA, F.; ALEXANDRE FILHO, L.; FORNARI, D.C.; OLIVEIRA, S.N. de. Bayesian genetic parameters for body weight and survival of Nile tilapia farmed in Brazil. Pesquisa Agropecuária Brasileira, v.46, p.33-43, 2011. DOI: 10.1590/S0100-204X2011000100005.

VAN TASSEL, C.P.; VAN VLECK, D.L. A manual for use of MTGSAM: a set of FORTRAN programs to apply Gibbs sampling to animal models for variance component estimation (DRAFT). Lincoln: Department of Agriculture Research Service, 1995. 86p.

Recebido em 22 de junho de 2011 e aprovado em 29 de outubro de 2012 